\title{
Multicellular spheroids of bone marrow stromal cells: a three-dimensional in vitro culture system for the study of hematopoietic cell migration
}

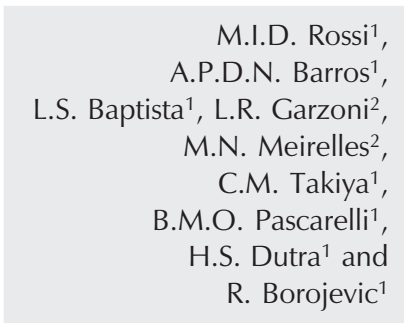

\author{
${ }^{1}$ Departamento de Histologia e Embriologia, \\ Instituto de Ciências Biomédicas, \\ Universidade Federal do Rio de Janeiro, Rio de Janeiro, RJ, Brasil \\ ${ }^{2}$ Laboratório de Ultra-estrutura Celular, \\ Departamento de Ultra-estrutura e Biologia Celular, Instituto Oswaldo Cruz, \\ Fiocruz, Rio de Janeiro, RJ, Brasil
}

\begin{abstract}
Correspondence
M.I.D. Rossi

Departamento de Histologia e

Embriologia, ICB, CCS, UFRJ

Bloco F, 2 andar

21941-970 Rio de Janeiro, RJ

Brasil

Fax: +55-21-2562-2467

E-mail: idrossi@hucff.ufrj.br

Presented at SIMEC 2004

(International Symposium

on Extracellular Matrix),

Angra dos Reis, RJ, Brazil,

September 27-30, 2004.

Research supported by FAPERJ (No. E-26/170.641/2004), CNPq, CAPES, and APABCAM, Brazil.

Received February 16, 2005

Accepted May 5, 2005

Cell fate decisions are governed by a complex interplay between cellautonomous signals and stimuli from the surrounding tissue. In vivo cells are connected to their neighbors and to the extracellular matrix forming a complex three-dimensional (3-D) microenvironment that is not reproduced in conventional in vitro systems. A large body of evidence indicates that mechanical tension applied to the cytoskeleton controls cell proliferation, differentiation and migration, suggesting that 3 -D in vitro culture systems that mimic the in vivo situation would reveal biological subtleties. In hematopoietic tissues, the microenvironment plays a crucial role in stem and progenitor cell survival, differentiation, proliferation, and migration. In adults, hematopoiesis takes place inside the bone marrow cavity where hematopoietic cells are intimately associated with a specialized three 3-D scaffold of stromal cell surfaces and extracellular matrix that comprise specific niches. The relationship between hematopoietic cells and their niches is highly dynamic. Under steady-state conditions, hematopoietic cells migrate within the marrow cavity and circulate in the bloodstream. The mechanisms underlying hematopoietic stem/progenitor cell homing and mobilization have been studied in animal models, since conventional two-dimensional (2-D) bone marrow cell cultures do not reproduce the complex $3-\mathrm{D}$ environment. In this review, we will highlight some of the mechanisms controlling hematopoietic cell migration and 3-D culture systems.
\end{abstract}

\section{Abstract}

\section{Introduction}

In mammalian tissues, cells are connected to each other and to a mesh of extracellular matrix (ECM) that forms a three-dimensional
Key words

- Multicellular spheroid

- Three-dimensional culture

- Cell migration

- Bone marrow
(3-D) scaffold. It is well established that cell contact with ECM, via integrins, affects many cellular functions such as proliferation, differentiation, migration, apoptosis, and cell shape (reviewed in Ref. 1). However, in 
order to study these cellular processes, cells are usually isolated from the tissues and grown in two-dimensional (2-D) culture systems. Unfortunately, this alters the interactions between cells and the ECM and the complex organization of integrins and cytoskeleton molecules, modifying cell shape. Since composition and organization of ECM as well as cell shape can markedly influence cell proliferation, differentiation, and migration (1-3), the degree to which the results obtained using 2-D cultures represent the in vivo situation should be considered with caution.

\section{Three-dimensional culture systems: revealing cellular subtleties}

Usually, cell differentiation is associated with changes in cell shape that are thought to arise by the modulation of integrin signaling pathways and cytoskeleton molecules $(1,3)$. However, there is increasing evidence that changes in cell shape themselves can actually affect cell fate decisions. Cell morphology influences proliferation (4), differentiation (5), gene expression (6), and migration (7). Recently, it was shown that cell shape drives the differentiation of human mesenchymal stem cells towards the adipogenic and osteogenic lineages. Shape-dependent control of cell differentiation was shown to be mediated by Rho small GTPase via ROCK-mediated cytoskeleton tension (2).

On this basis, it should be considered that the dramatic change in cell morphology that occurs when they are grown on a plastic surface could affect cell behavior. Although being conveniently used for the maintenance of cells and for biological studies, these conventional culture systems are 2-D, and impose highly unnatural geometric and mechanical constraints, with an artificial polarization and spreading of many types of cells. So, it is not surprising that investigators have recently reported striking differences in results obtained using 2-D cultures as opposed to those obtained in vivo and in 3-D cultures. Comparing the behavior of fibroblasts in 2D versus 3-D cultures, Cukierman and colleagues (8) observed that in 3-D, the cells moved and divided more quickly, and assumed the characteristic asymmetric shape that fibroblasts have in living tissues. Furthermore, these investigators noticed that in $3-\mathrm{D}$, as well as in vivo, fibroblast interaction with ECM did not involve the development of focal adhesions or the phosphorylation of focal adhesion tyrosine kinases. Recently, it was shown that in tissue cultures in plastic plates, fibroblasts show a redistribution of anchoring receptors to their ventral surface and that the lack of dorsal receptor anchorage created an imbalance that would cause cells to spread out (9). This imbalance also affected cell migration, cytoskeleton organization, and the development of focal adhesions.

Striking differences in cell behavior in 2D versus 3-D culture systems were also described by the group of Dr. Bissel, who observed that incubation of mammary carcinoma cells with antibody against 31 -integrin inhibited cell growth and reversed the malignant phenotype in vivo and in 3-D cultures. However, these effects were not observed when the cells were cultured as monolayers (10). The cell growth arrest and acinus formation were due to a bidirectional cross-modulation of 31 -integrin and epidermal growth factor receptor signaling via the mitogen-activated protein kinase pathway. More importantly, this reciprocal modulation did not occur in monolayers, in which qualitative changes in epidermal growth factor receptor and integrin signaling pathway and their coupling to the mitogen-activated protein kinase pathway were observed (11).

It is well established that tumor cell invasion and migration involves adhesion to ECM substrate coordinated with proteolytic cleavage by matrix metalloproteinases (MMPs), resulting in the degradation and remodeling of interstitial tissue barriers. However, in 
vivo targeting of MMP function by protease inhibitors showed unexpectedly weak benefit in some animal tumors (reviewed in Ref. 12). A mechanism that explains the failure in inhibiting tumor cell invasion by blocking MMP activity has been recently proposed. Using a 3-D fibrillar collagen-rich matrix culture, Wolf and colleagues (13) showed a switch to a nonproteolytic "amoeboid" migration after abrogation of the ECM-proteolysis capacity of a fibrosarcoma cell line. This "amoeboid" movement was also observed in vivo, after blocking MMP activity.

This compelling evidence of the influence of cell culture conditions on cell behavior suggests that 3-D culture systems can provide a better model for what happens in vivo and would be an intermediate model between conventional in vitro cell culture systems and experimental animal models.

\section{Multicellular spheroids: a 3-D in vitro culture system}

Multicellular spheroids, a 3-D cell culture system, have been widely used over the past four decades as in vitro systems to study the mechanisms underlying organogenesis, tumor cell response to therapy, and epithelial-mesenchymal cell interactions in tumors. These spherical reaggregated cell cultures were first introduced as an approach to the understanding of morphogenesis. Since spontaneous cell-cell interactions are developed in a non-preorganized scaffold from dispersed cells of a particular tissue, this method allows the investigation of basic principles of tissue formation. Later on, malignant cellular reaggregates, the multicellular tumor spheroids, were developed from solid tumors and were proven to be a useful model for systematic studies of tumor cell response to therapy. Nowadays, the spheroid approach is seen as a scaffold-free artificial microtissue useful for tissue engineering research. In contrast to conventional monolayer cultures, these cellular aggregates would allow the development of a more or less complex 3-D cell-to-cell and cell-to-ECM interactions, similar to the corresponding tissues in vivo (reviewed in Refs. 14-19).

Technically, a single cell suspension is obtained by enzymatic and/or mechanical dissociation and reaggregated as 3-D spheres by a variety of methods. The main issue is to provide culture conditions such that the adhesive forces between cells are greater than those for the substrate on which they are plated. Spheroids can be developed by: i) plating cells in gyratory shakers, roller flasks, or spinner flasks that continuously rotate preventing cellular adherence to the vessel walls; ii) simply coating the tissue culture surfaces with a thin layer of agarose or another nonadhesive substance. Under these conditions, cells would not be able to adhere to the plastic surface of culture flasks and many cell types would undergo homotypic aggregation; iii) using the hanging drop culture method, a type of culture originally developed for raising embryoid bodies, or iv) centrifuge compression of cells to the bottom of conical tubes. The pellet is gently raised into the culture medium to avoid disaggregation and cultured $(16,17,19)$.

Besides spheroid monocultures, spheroid co-cultures can be developed and this socalled 'hetero-spheroids' yield a similar or better organotypic differentiation pattern than spheroid monocultures. Spheroid co-culture of tumor and endothelial cells as well as tumor and tumor-derived stromal cells brought some insights into the mechanism of angiogenesis, invasion, and metastasis (14-16). Using a spheroid invasion assay, Brabletz and collaborators (20) showed that nuclear $\beta$-catenin was found exclusively in dedifferentiated mesenchyme-like carcinoma cells at the invasive front. Strikingly, as it happens in the primary tumors, B-catenin was localized to the membrane and cytoplasm of epithelial tumor cells. A correlation between the nuclear localization of B-catenin and lack of E-cadherin as well as prolifera- 
tive activity was observed in both the primary tumor and the spheroid hetero-culture. In 1998, Korf and Augustin (21) described the development of an endothelial cell spheroid culture as an in vitro model for angiogenesis. Usually, co-culture spheroids of endothelial cells with smooth muscle cells or osteoblast organized spontaneously in a core of smooth muscle cells or osteoblasts and a surface of endothelial cells $(22,23)$. Recently, a hetero-spheroid model based on the co-culture of isolated endothelial cells with a pre-formed multicellular spheroid allowed the establishment of a vascular network that mimics tumor angiogenesis (24).

\section{Bone marrow microenvironment and hematopoietic stem cell homing}

In adults, hematopoiesis takes place in the extravascular spaces of bone marrow between the venous sinuses and in close contact with a heterogeneous stromal cell population $(25,26)$. Bone marrow stromal tissue is a 3-D continuum of cell surfaces comprising cells of non-hematopoietic origin like reticular cells, adipocytes, osteogenic cells near the bone surfaces, vascular endothelial cells and smooth muscle cells in vessel walls, as well as cells of hematopoietic origin, mostly macrophages $(25,26)$. Within the marrow environment, hematopoietic cells exhibit distinctive and lineagespecific spatial locations $(25,27)$ and it has been assumed that these specific cellular interactions create special microenvironments known as niches. Consistent with this, it has been shown that hematopoietic stem cells (HSC) are located near the subendosteal region (27-29), in contact with osteogenic cells that are key regulators of these HSC niches $(30,31)$. It should be emphasized that members of our group recently showed that the subendosteal region harbors stromal cells that differ in their capacity to support HSC self-renewal and proliferation (32). Differentiation of HSCs and their progeny occurs while they migrate toward the central area of the marrow cavity and more differentiated hematopoietic cells exit the marrow into the blood through the central sinus walls $(29,33$, 34). Actually, it has been shown that under steady-state conditions HSCs also transit between marrow and blood at a higher frequency than previously thought (35), suggesting that the release of HSCs into the circulation and their homing back to the marrow are important events in the homeostasis of hematopoietic tissue.

Circulating HSCs seed very rapidly the subendosteal region of the marrow $(29,36)$ but how they do it is still a matter of debate. Nilsson and colleagues (29) have observed that $1 \mathrm{~h}$ after transplantation, the majority of donor HSCs were located in the central region, but after $5 \mathrm{~h}$ most of them were already in the subendosteal region. They proposed a model whereby HSCs transendothelially migrate from the venous sinuses after having passed through the bone cortex, with some transendothelial migration occurring in the subendosteal region. According to this model, most HSCs, after entering the bone marrow environment, should migrate through it until they reach their niches in the subendosteal region. However, Askenazy and colleagues (36) have reported the presence of clusters of donor HSCs in the subendosteal region 3 to $5 \mathrm{~min}$ after transplant, calling attention to the fact that during the first hour after transplantation a great motility of donor HSCs within the marrow environment was noticed. Interestingly, a few days after transplantation, some cells were observed in the more central regions of marrow (36), suggesting that the exit of HSCs from marrow to the blood also occurs through the venous sinuses. This means that HSCs must leave their niches near the endosteum and migrate toward the central sinus.

The mechanisms that allow the physiological circulation of HSCs are not clear, but some insights have emerged from the enforced migration, also known as mobili- 
zation, of HSCs from marrow to the blood, that can be induced by a wide variety of molecules, including cytokines such as granulocyte-colony stimulating factor (GCSF) and chemotherapeutic agents (reviewed in Refs. 37,38). HSC mobilization should be seen as a multistep process in which disruption of adhesion to bone marrow stromal cells and ECM needs to be coordinated with cytoskeleton reorganization that would drive cell migration along the marrow axis toward the venous sinuses. Among the molecules that are involved in cell-to-cell contact, a member of the integrin family, the very late activation antigen-4/CD $49 d$ that interacts mostly with vascular cell adhesion molecule-1/CD106 has been found to be an important mediator of progenitor cell adhesion to marrow stromal cells in vitro (39), as well as mobilization and homing of HSCs (38, 40,41).

Chemokines are also key players in HSC mobilization and homing. It has been shown that homing and retention of human and murine HSCs are dependent on the CXC chemokine ligand 12, also known as stromal cell-derived factor-1 (SDF-1) $(42,43)$. This prompted scientists to investigate the role of SDF-1 and CXCR4 in HSC mobilization. Plasma elevation of SDF-1, induced by intravenous injection of adenoviral vector expressing SDF-1, resulted in HSC mobilization (44). The role of the CXCR4/SDF-1 axis in human HSC mobilization was recently supported by the observation that GCSF reduces the expression of SDF-1 within the bone marrow, without affecting protein levels in plasma (45). Taken together, these results suggest that HSC homing and mobilization are driven by an SDF-1 gradient concentration that provides directional cues for HSCs.

Protein cleavage also seems to be an important event in HSC mobilization. An increase in bone marrow contents of proteolytic enzymes (neutrophil elastases and MMPs) is observed after G-CSF treatment and many molecules implicated in HSC homing and mobilization, like SDF-1 and vascular cell adhesion molecule-1, are their substrate $(38,40)$. Interestingly, G-CSF induces osteoclast activation and bone resorption, resulting in the release of soluble calcium from the endosteum that causes detachment of HSCs from fibronectin (46). Osteoclasts also play a role in bone remodeling, which gives rise to the hypothesis that the bone marrow niches for HSCs are actually very dynamic. Under steady-state conditions, the remodeling of HSC niches could be involved in the physiological circulation of HSCs.

Cell adhesion, migration, and movement in response to chemoattractants involve cytoskeleton reorganization that is mostly regulated by Rho GTPases. The role of Rho GTPase proteins in HSC migration has only recently been investigated. Rho-related GTPases, including Rho, Rac, and Cdc42, seem to be specifically involved in HSC adhesion, migration, and proliferation (47, 48). Rac2-deficient HSCs showed a decrease in $\beta 1$ integrin-mediated adhesion with an increase in in vitro motility in response to SDF-1. This hypermotility of HSCs was related to a compensatory activation of $\mathrm{Cdc} 42$. Morphological changes, such as uniformly longer filopodia, F-actin expression, and spike-like projections, were also observed in Rac2 $^{-/-}$cells after SDF-1 stimulation (47). The importance and specificity of Rho-related GTPases in HSC properties were confirmed by the observation that Rac1 predominantly regulates cell cycle progression, while Rac2 regulates apoptosis and F-actin assembly in response to SDF-1 (48). Furthermore, a cross-modulation of Rho-GTPases, integrin and chemokine signaling pathways has been reported in lymphocytes (49) and hematopoietic progenitor cells (50).

In view of these considerations, we should think of the bone marrow microenvironment as a very dynamic one with hematopoietic cells constantly rolling over a 3-D continuum scaffold of stromal cell surfaces and ECM. 
This highly organized 3-D microenvironment is not reproduced in conventional cell cultures.

\section{Bone marrow stromal cell spheroids as an in vitro model to study hematopoietic cell migration} widely used to study solid tumors, tissue regeneration, and bioengineering, no such application have been used for the study of the hematopoietic system. Bone marrow stromal cell 3-D culture systems have been developed mostly for bone tissue engineering purposes. In order to favor osteoblastic differentiation, bone marrow stromal cells were grown on a 3-D scaffold made of irradiated bones or biomaterials $(51,52)$. Scaffold-free cellular aggregates of human bone marrow stromal cells were developed as an assay for chondrogenesis $(53,54)$ and recently as an in vitro system for endochondral ossification (55). Preliminary results from our laboratory have shown that human bone marrow stromal cell aggregates formed a network of cells and fibronectin that reproduced a 3-D quiescent environment. The diameter of these spheroids correlated directly with the number of plated cells (Figure 1). In the absence of an inductive medium, the cells maintained the expression of smooth muscle $\alpha$ actin, without forming the stress fibers observed in monolayers (Barros et al., unpublished results).

Figure 1. Bone marrow stromal cell spheroids. $A$, Bone marrow stromal cells plated onto a nonadhesive surface form spherical aggregates with a dense homogeneous appearance. Phase contrast. Bar $=100 \mu \mathrm{m}$. $B$, The size of the spheroids corresponds to the number of cells initially plated. Data are reported as the average size of spheroid \pm SD. The diameter of 6 spheroids per cell number was individually measured with an inverted microscope equipped with a digital camera.

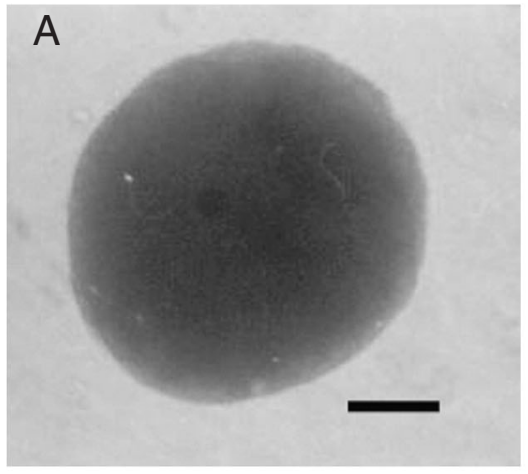

Although 3-D culture systems have been

In 2002, the group of Dr. Kunz-Shughart adapted a spheroid co-culture model developed to investigate reciprocal tumor-immune cell interactions to study the mechanisms of HSC locomotion in the bone marrow microenvironment (56). The authors showed that human hematopoietic progenitor cells isolated from umbilical cord blood were able to interact with 3-D aggregates of a murine bone marrow stromal cell line but, in contrast to what has been reported for animal models, migration of progenitor cells in this in vitro system was independent of $B 1$ integrins and CXCR4, but was significantly impaired by inhibitors of Rho family small GTPases. The authors observed that about $20 \%$ of the cells were able to migrate after 48 $\mathrm{h}$ of co-culture without signs of saturation. Preliminary results from our laboratory showed that $\mathrm{CB}$ progenitor cells $\left(\mathrm{CD} 34^{+}\right.$ CD45 ${ }^{\mathrm{Lo}}$ ) were capable of invading human bone marrow stromal cell aggregates. In agreement with Bug and colleagues (56), migration of $\mathrm{CB} \mathrm{CD} 34^{+}$cells into the spheroids was shown to depend on GTPases. However, a peak was observed after $24 \mathrm{~h}$ of co-culture. We are also testing this culture system as a model for studying migration and invasion of transformed hematopoietic cells.

\section{Concluding remarks}

Living tissues are 3-D structures where cells are bound together by a mesh of ECM.

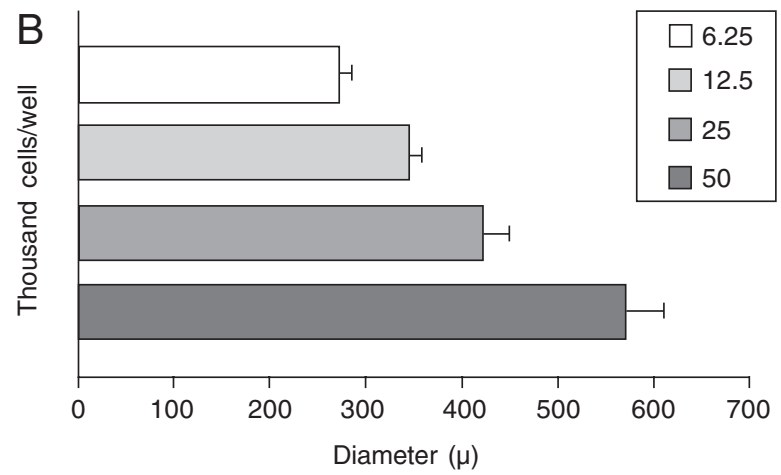


Cell-to-cell contact, as well as contact with ECM affect many cellular processes (shape, differentiation, proliferation, migration, and apoptosis). This complex organization is disrupted when cells are cultured onto a plastic tissue flask, which could give rise to misleading results. Nowadays, scientists are developing 3-D culture systems that mimic more or less the in vivo situation. These culture systems, when compared to conventional cultures, are revealing subtle, and sometimes striking, differences in cell behavior, suggesting that scientists in different fields should take advantage of 3-D culture systems to explore cell function in a complex milieu.
Hematopoiesis is controlled by a complex interplay between hematopoietic cells and a 3-D microenvironment. Cellular interactions in this environment are very dynamic and involve cross-modulation of adhesion molecules, chemokines, cytokines, proteolytic enzymes, and regulators of cytoskeleton organization such as members of the Ras superfamily. However, the mechanisms of hematopoietic cell migration are not fully understood. We suggest that the development of in vitro models that allow complex 3-D cell-cell and cell-ECM interactions will help to clarify and depict these mechanisms.

\section{References}

1. Boudreau NJ \& Jones PL (1999). Extracellular matrix and integrin signaling: the shape of things to come. Biochemical Journal, 339: 481-488.

2. McBeath R, Pirone DM, Nelson CM et al. (2004). Cell shape, cytoskeletal tension, and RhoA regulate stem cell lineage commitment. Developmental Cell, 6: 483-495.

3. Walpita D \& Hay E (2002). Studying actin-dependent processes in tissue culture. Nature Reviews. Molecular Cell Biology, 3: 137-141.

4. Chen CS, Mrksich M, Huang S et al. (1997). Geometric control of cell life and death. Science, 276: 1425-1428.

5. Lelièvre SA, Weaver VM, Nickerson JA et al. (1998). Tissue phenotype depends on reciprocal interactions between the extracellular matrix and the structural organization of the nucleus. Proceedings of the National Academy of Sciences, USA, 95: 14711-14716.

6. Thomas $\mathrm{CH}$, Collier JH, Sfeir CS et al. (2002). Engineering gene expression and protein synthesis by modulation of nuclear shape. Proceedings of the National Academy of Sciences, USA, 99: 19721977.

7. Jiang X, Bruzewicz DA, Wong AP et al. (2005). Directing cell migration with asymmetric micropatterns. Proceedings of the National Academy of Sciences, USA, 102: 975-978.

8. Cukierman E, Pankov R, Stevens DR et al. (2001). Taking cellmatrix to the third dimension. Science, 294: 1708-1712.

9. Beningo KA, Dembo M \& Wang Y-L (2004). Responses of fibroblasts to anchorage of dorsal extracellular matrix receptors. Proceedings of the National Academy of Sciences, USA, 52: 1802418029.

10. Weaver VM, Petersen OW, Wang F et al. (1997). Reversion of the malignant phenotype of human breast cells in three-dimensional culture and in vivo by integrin blocking antibodies. Journal of Cell Biology, 137: 231-245.

11. Wang F, Weaver VM, Petersen OW et al. (1998). Reciprocal interactions between $B 1$-integrin and epidermal growth factor receptor in three-dimensional basement membrane breast cultures: a different perspective in epithelial biology. Proceedings of the National Academy of Sciences, USA, 95: 14821-14826.

12. Coussens LM, Fingleton B \& Matrisian LM (2002). Matrix metalloproteinase inhibitors and cancer: trials and tribulations. Science, 295: 2387-2392.

13. Wolf K, Mazo I, Leung $\mathrm{H}$ et al. (2003). Compensation mechanism in tumor cell migration: mesenchymal-amoeboid transition after blocking of pericellular proteolysis. Journal of Cell Biology, 160: 267-277.

14. Mueller-Klieser W (1997). Three-dimensional cell cultures: from molecular mechanisms to clinical applications. American Journal of Physiology, 273: C1109-C1123.

15. Kunz-Shughart LA, Kreutz M \& Knuechel R (1998). Multicellular spheroids: a three-dimensional in vitro culture system to study tumour biology. International Journal of Experimental Pathology, 79: 1-23.

16. Kunz-Shughart LA (1999). Multicellular tumor spheroids: intermediates between monolayer culture and in vivo tumor. Cell Biology International, 23: 157-161.

17. Bates RC, Edwards NS \& Yates JD (2000). Spheroids and cell survival. Critical Reviews in Oncology/Hematology, 36: 61-74.

18. Layer PG, Robitzki A, Rothermel A et al. (2002). Of layers and spheres: the reaggregate approach in tissue engineering. Trends in Neurosciences, 25: 131-134.

19. Kelm JM \& Fussenegger M (2004). Microscale tissue engineering using gravity-enforced cell assembly. Trends in Biotechnology, 22: 195-202.

20. Brabletz T, Jung A, Reu S et al. (2001). Variable B-catenin expression in colorectal cancers indicates tumor progression driven by the tumor environment. Proceedings of the National Academy of Sciences, USA, 98: 10356-10361.

21. Korf T \& Augustin HG (1998). Integration of endothelial cells in multicellular spheroids prevents apoptosis and induces differentiation. Journal of Cell Biology, 143: 1341-1352.

22. Korf T, Kimmina S, Martiny-Baron G et al. (2001). Blood vessel 
maturation in a 3-dimensional spheroidal coculture model: direct contact with smooth muscle cells regulates endothelial cell quiescence and abrogates VEGF responsiveness. FASEB Journal, 15: 447-457.

23. Stahl A, Wenger A, Weber $\mathrm{H}$ et al. (2004). Bi-directional cell contactdependent regulation of gene expression between endothelial cells and osteoblasts in a three-dimensional spheroidal coculture model. Biochemical and Biophysical Research Communications, 322: 684692.

24. Timmins NE, Dietmair S \& Nielsen LK (2004). Hanging-drop multicellular spheroids as a model of tumour angiogenesis. Angiogenesis, 7: 97-103.

25. Weiss $L$ (1976). The hematopoietic microenvironment of the bone marrow: an ultrastructural study of the stroma in rats. Anatomical Record, 186: 161-184.

26. Short B, Brouard N, Occhiodoro-Scott T et al. (2003). Mesenchymal stem cells. Archives of Medical Research, 34: 565-571.

27. Lambertsen RH \& Weiss L (1984). A model of intramedullary hematopoietic microenvironments based on stereologic study of the distribution of endocloned marrow colonies. Blood, 63: 287-297.

28. Gong JK (1978). Endosteal marrow: a rich source of hematopoietic stem cells. Science, 199: 1443-1445.

29. Nilsson SK, Johnston HM \& Coverdale JA (2001). Spatial localization of transplanted hematopoietic stem cells: inferences for the localization of stem cell niches. Blood, 97: 2293-2299.

30. Calvi LM, Adams GB, Welbrecht KW et al. (2003). Osteoblastic cells regulate the hematopoietic stem cell niche. Nature, 425: 841-846.

31. Zhang J, Niu C, Ye L et al. (2003). Identification of the haematopoietic stem cell niche and control of the niche size. Nature, 425: 836841.

32. Balduino A, Hurtado SP, Frazão P et al. (2005). Bone marrow subendosteal microenvironment harbours functionally distinct haemosupportive stromal cell populations. Cell and Tissue Research, 319: 255-266.

33. Jacobsen K \& Osmond DG (1990). Microenvironment organization and stromal cell associations of B lymphocyte precursor cells in mouse bone marrow. European Journal of Immunology, 20: 23952404.

34. Tokoyoda K, Egawa T, Sugiyama T et al. (2004). Cellular niches controlling B lymphocyte behavior within bone marrow during development. Immunity, 20: 707-718.

35. Wright DE, Wagers AJ, Gulati AP et al. (2001). Physiological migration of hematopoietic stem and progenitor cells. Science, 294: 19331935.

36. Askenazy N, Zorina T, Farkas DL et al. (2002). Transplanted hematopoietic cells seed in clusters in recipient bone marrow in vivo. Stem Cells, 20: 301-310.

37. Lapidot T \& Petit I (2002). Current understanding of stem cell mobilization: the roles of chemokines, proteolytic enzymes, adhesion molecules, cytokines, and stromal cells. Experimental Hematology, 30: $973-981$

38. Papayannopoulo T (2004). Current mechanistic scenarios in hematopoietic stem/progenitor cell mobilization. Blood, 103: 15801585.

39. Miyake K, Weissman IL, Greenberger JS et al. (1991). Evidence for a role of the integrin VLA-4 in lympho-hemopoiesis. Journal of Experimental Medicine, 173: 599-607.

40. Lévesque JP, Takamatsu Y, Nilsson SK et al. (2001). Vascular cell adhesion molecule-1 (CD106) is cleaved by neutrophil proteases in the bone marrow following hematopoietic progenitor cell mobilization by granulocyte colony-stimulating factor. Blood, 98: 1289-1297.

41. Papayannopoulo T, Priestley GV, Nakamoto B et al. (2001). Molecular pathways in bone marrow homing: dominant role of $\alpha_{4} \beta_{1}$ over $B_{2}$-integrins and selectins. Blood, 98: 2403-2411.

42. Nagasawa T, Hirota S, Tachibana K et al. (1996). Defects of B-cell lymphopoiesis and bone-marrow myelopoiesis in mice lacking the CXC chemokine PBSF/SDF-1. Nature, 382: 635-638.

43. Peled A, Petit I, Kollet O et al. (1999). Dependence of human stem cell engraftment and repopulation of NOD/SCID mice on CXCR4. Science, 283: 845-848.

44. Hattori K, Heissig B, Tashiro K et al. (2001). Plasma elevation of stromal cell-derived factor-1 induces mobilization of mature and immature hematopoietic progenitor and stem cells. Blood, 97: 33543360.

45. Petit I, Szyper-Kravitz M, Nagler A et al. (2002). G-CSF induces stem cell mobilization by decreasing bone marrow SDF-1 and upregulating CXCR4. Nature Immunology, 3: 687-694.

46. Takamatsu Y, Simmons PJ, Moore RJ et al. (1998). Osteoclastmediated bone resorption is stimulated during short-term administration of granulocyte colony-stimulating factor but is not responsible for hematopoietic progenitor cell mobilization. Blood, 92: 3465-3473.

47. Yang F-C, Atkinson SJ, Gu Y et al. (2001). Rac and Cdc42 GTPases control hematopoietic stem cell shape, adhesion, migration, and mobilization. Proceedings of the National Academy of Sciences, USA, 98: 5614-5618.

48. Gu Y, Filippi M-D, Cancelas JA et al. (2003). Hematopoietic cell regulation by Rac1 and Rac2 guanosine triphosphatases. Science, 302: 445-449

49. Weber KSC, Ostermann G, Zernecke A et al. (2001). Dual role of HRas in regulation of lymphocyte function antigen- 1 activity by stromal cell-derived factor $1 \alpha$ : implications for leukocyte transmigration. Molecular Biology of the Cell, 12: 3074-3086.

50. Henschler R, Piiper A, Bistrian R et al. (2003). SDF-1 $\alpha$-induced intracellular calcium transient involves Rho GTPase signalling and is required for migration of hematopoietic progenitor cells. Biochemical and Biophysical Research Communications, 311: 1067-1071.

51. Niemeyer P, Krause U, Fellenberg J et al. (2004). Evaluation of mineralized collagen and alpha-tricalcium phosphate as scaffolds for tissue engineering of bone using human mesenchymal stem cells. Cells Tissues Organs, 177: 68-78.

52. Yang XB, Bhatnagar RS \& Oreffo RO (2004). Biomimetic collagen scaffolds for human bone cell growth and differentiation. Tissue Engineering, 10: 1148-1159.

53. Johnstone B, Hering TM, Caplan Al et al. (1998). In vitro chondrogenesis of bone marrow-derived mesenchymal progenitor cells. Experimental Cell Research, 238: 265-272.

54. Sekiya I, Vuoristo JT, Larson BL et al. (2001). In vitro cartilage formation by human adult stem cells from bone marrow stroma defines the sequence of cellular and molecular events during chondrogenesis. Proceedings of the National Academy of Sciences, USA, 99: 4397-4402.

55. Muraglia A, Corsi A, Riminucci M et al. (2003). Formation of a chondro-osseous rudiment in micromass cultures of human bonemarrow stromal cells. Journal of Cell Science, 116: 2949-2955.

56. Bug G, Rossmanith T, Henschler R et al. (2002). Rho family small GTPases control migration of hematopoietic progenitor cells into multicellular spheroids of bone marrow stroma cells. Journal of Leukocyte Biology, 72: 837-845. 